\title{
Extensive shared polymorphism at non-MHC immune genes in recently diverged North American prairie grouse
}

\author{
Piotr Minias ${ }^{1}$ - Zachary W. Bateson ${ }^{2} \cdot$ Linda A. Whittingham ${ }^{2}$ - Jeff A. Johnson ${ }^{3}$. \\ Sara Oyler-McCance ${ }^{4} \cdot$ Peter O. Dunn ${ }^{2}$
}

Received: 25 January 2017 / Accepted: 25 July 2017 / Published online: 2 August 2017

(C) The Author(s) 2017. This article is an open access publication

\begin{abstract}
Gene polymorphisms shared between recently diverged species are thought to be widespread and most commonly reflect introgression from hybridization or retention of ancestral polymorphism through incomplete lineage sorting. Shared genetic diversity resulting from incomplete lineage sorting is usually maintained for a relatively short period of time, but under strong balancing selection it may persist for millions of years beyond species divergence (balanced trans-species polymorphism), as in the case of the major histocompatibility complex (MHC) genes. However, balancing selection is much less likely to act on non-MHC immune genes. The aim of this study was to investigate the patterns of shared polymorphism and selection at non-MHC immune genes in five grouse species from Centrocercus and Tympanuchus genera. For this purpose, we genotyped five non-MHC immune genes that do not interact directly with pathogens, but are involved in signaling and regulate immune cell growth. In
\end{abstract}

Electronic supplementary material The online version of this article (doi:10.1007/s00251-017-1024-4) contains supplementary material, which is available to authorized users.

Piotr Minias

pminias@op.pl

1 Department of Biodiversity Studies and Bioeducation, Faculty of Biology and Environmental Protection, University of Łódź, Banacha 1/3, 90-237 Łódź, Poland

2 Behavioral and Molecular Ecology Group, Department of Biological Sciences, University of Wisconsin-Milwaukee, Milwaukee, WI, USA

3 Department of Biological Sciences, Institute of Applied Sciences, University of North Texas, Denton, TX, USA

4 Fort Collins Science Center, US Geological Survey, Ft. Collins, CO, USA contrast to previous studies with MHC, we found no evidence for balancing selection or balanced trans-species polymorphism among the non-MHC immune genes. No haplotypes were shared between genera and in most cases more similar allelic variants sorted by genus. Between species within genera, however, we found extensive shared polymorphism, which was most likely attributable to introgression or incomplete lineage sorting following recent divergence and large ancestral effective population size (i.e., weak genetic drift). Our study suggests that North American prairie grouse may have attained relatively low degree of reciprocal monophyly at nuclear loci and reinforces the rarity of balancing selection in non-MHC immune genes.

Keywords Ancestral polymorphism · Grouse · Immune genes $\cdot$ Introgression $\cdot$ Reciprocal monophyly $\cdot$ Selection

\section{Introduction}

Genetic variation shared between recently diverged taxa is most commonly explained with retention of ancestral polymorphisms or introgressive hybridization (Donnelly et al. 2004; Zhou et al. 2017). The first mechanism is consistent with incomplete lineage sorting when ancestral allelic variants are maintained in both descendant species following neutral expectation. Incomplete lineage sorting is widespread and likely to persist for some period of time after species divergence, depending on the strength of drift (negatively correlated with the effective population size) and the mutation rate for a particular set of loci (Hudson and Coyne 2002; Hedrick 2013). Similar patterns of shared genetic diversity are also produced by introgression, i.e., the horizontal transfer of allelic lineages, which may either contribute to the adaptive genetic variation of the recipient species or may be nonadaptive (detrimental or neutral). Recently, it has been 
suggested that introgression may be much more common in vertebrates than previously thought (Rheindt and Edwards 2011; Hedrick 2013), and there is accumulating evidence for the role of introgression in generating variation at immune genes of different animal taxa (Parmakelis et al. 2008; Nadachowska-Brzyska et al. 2012; Grossen et al. 2014).

Assuming that multiple ancestral allelic variants are adaptive, their polymorphism shared by descendant species may be maintained by balancing selection for longer periods of time beyond neutral expectations, which is often referred to as balanced trans-species polymorphism (TSP). However, shared polymorphism can also evolve independently in two or more lineages from different ancestral states as a result of adaptation to similar selective pressures (convergent evolution). Among the immune genes, balanced TSP and convergent evolution have been almost exclusively reported for the major histocompatibility complex (MHC), where balancing selection can maintain ancestral allelic variants for millions of years following divergence (Aguilar and Garza 2007; Kamath and Getz 2011) and exposure to similar pathogen pressure can produce convergence at the amino acid sequence level (Yeager et al. 1997; Kriener et al. 2000; Srithayakumar et al. 2012). So far, molecular evidence for balanced TSP and convergence at non-MHC immune genes are very limited (Leulier and Lemaitre 2008; Téšický and Vinkler 2015).

The aim of this study was to investigate the patterns of shared polymorphism at non-MHC immune genes in five grouse species from two closely-related genera, Centrocercus and Tympanuchus (Drovetski 2003; Galla and Johnson 2015; Stein et al. 2015). We examined five immune genes that do not directly interact with pathogens, but are involved in signaling and regulating immune cell growth: chicken B cell marker 6 (ChB6 or $\mathrm{Bu}-1$ ), inhibitor of apoptosis protein-1 (IAP-1), interleukin-2 (IL-2), transforming growth factor $\beta 3$ (TGF- $\beta 3$ ), and tumor necrosis factor-related apoptosis inducing ligand-like protein (TRAIL-like). Polymorphisms at these genes were associated with general mortality and growth (Ye et al. 2006), as well as with specific fitnessrelated immune traits in a close relative of grouse, the domestic chicken Gallus gallus. Polymorphisms at ChB6 were associated with regression of Rous sarcoma and resistance to Marek's disease (Gilmour et al. 1986; Taylor et al. 2016), while IAP-1 polymorphism showed associations with Salmonella enteritidis burden in the spleen (Liu and Lamont 2003) and antibody response kinetics to Brucella abortus (Zhou and Lamont 2003). Fitness associations for three of these genes have been reported for synonymous SNPs and SNPs in nontranslated genic regions, suggesting their linkage to functional polymorphisms. For example, a synonymous and an intronic polymorphism at TRAIL-like and
TGF- $\beta 3$ genes, respectively, were associated with bacterial load in the cecum and spleen contents of chickens (Malek and Lamont 2003).

Based on fossil-calibrated gene trees using both nuclear and mtDNA loci, the divergence between Centrocercus and Tympanuchus is estimated at ca. 6 to 8 million years, and species divergence within the two genera was within the last million years (Galla and Johnson 2015; Stein et al. 2015; Persons et al. 2016). While no information exists on the occurrence of Centrocercus hybrids (Young et al. 2015), hybridization has been widely documented between Tympanuchus species in areas of geographic overlap (Bain and Farley 2002; Augustine and Trauba 2015; Oyler-McCance et al. 2016) and hybrids appear to persist across generations due to sex-biased introgression (Galla and Johnson 2015). In contrast, Centrocercus-Tympanuchus hybrids are rare, and backcrossing is unlikely due to behavioral post-zygotic isolation (Aldridge et al. 2001; see also Augustine and Trauba 2015). Thus, we expected extensive shared polymorphisms within each of the two grouse genera, Tympanuchus and Centrocercus, resulting from incomplete lineage sorting and/ or introgression and low or absent levels of shared polymorphism between genera due to accumulating mutations and drift.

\section{Material and methods}

\section{Sample collection}

Samples were collected for five species of North American grouse: Centrocercus minimus (Gunnison sage-grouse), C. urophasianus (greater sage-grouse), Tympanuchus phasianellus (sharp-tailed grouse), Tympanuchus pallidicinctus (lesser prairie-chicken), and Tympanuchus cupido (greater prairie-chicken). These species are the only members of Centrocercus and Tympanuchus genera and together with two Dendragapus species form a monophyletic clade within Tetraoninae (Drovetski 2003; Stein et al. 2015). Blood and feather samples for the greater prairie-chicken were collected between 1990 and 2006 from six populations (Kansas, Minnesota, Missouri, Nebraska, Texas, and Wisconsin; Fig. 1). In total, 247 individuals were sampled and previously genotyped at all five immune genes by Bollmer et al. (2011). For the remaining species, blood and tissue samples (30 individuals per species) were collected between 1997 and 2011 in the following states: Colorado (C. minimus, C. urophasianus), Nevada and Utah (C. urophasianus), Wyoming (C. urophasianus, $T$. phasianellus), North Dakota (T. phasianellus), and Kansas (T. pallidicinctus) (Fig. 1). Protocols used for DNA extraction are described elsewhere (Bellinger et al. 2003; OylerMcCance et al. 2005; Galla and Johnson 2015). 


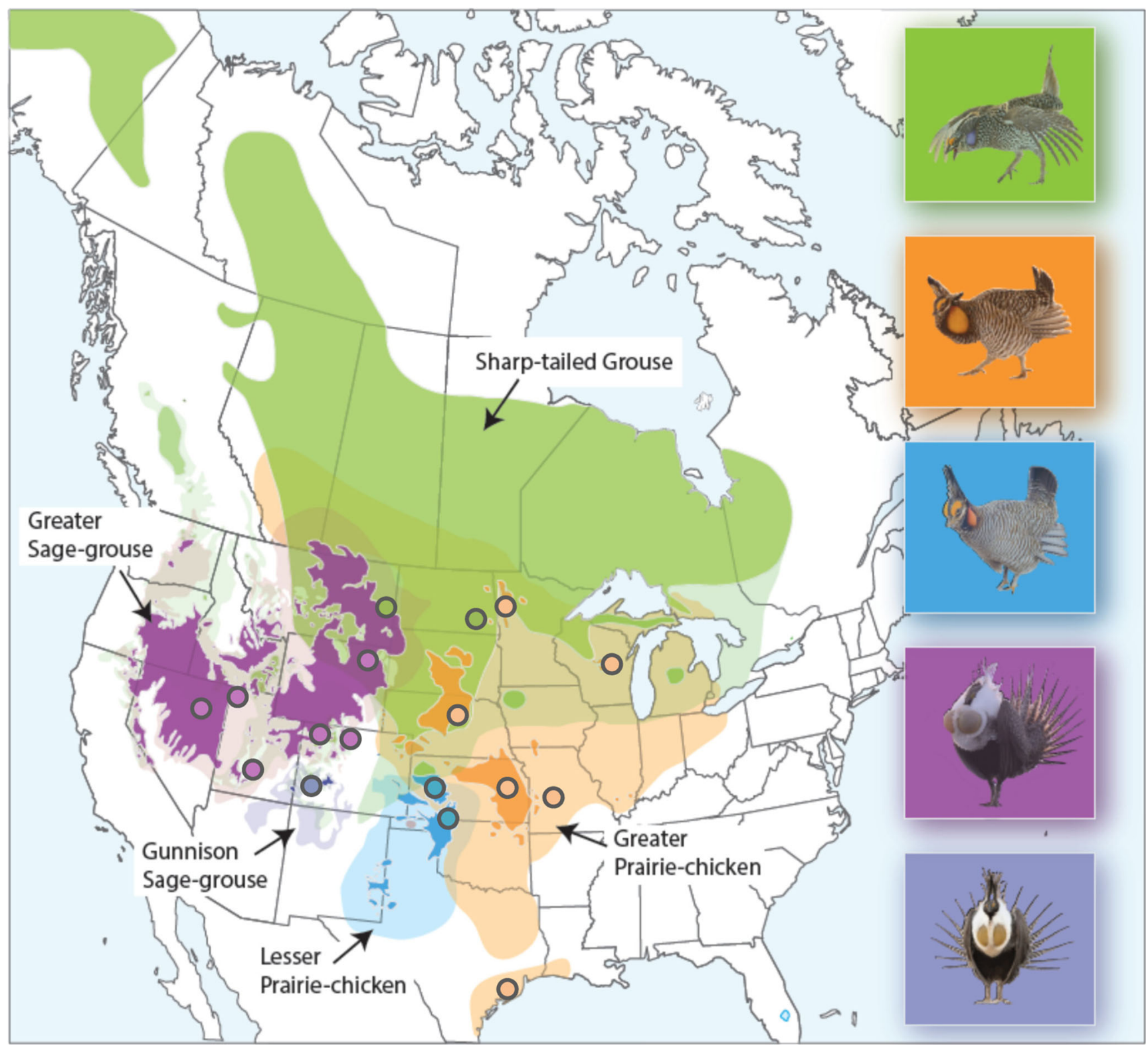

Fig. 1 Contemporary (full color) and historic (semi-transparent color) distributions of Centrocercus (Gunnison sage-grouse C. minimus - dark blue, greater sage-grouse C. urophasianus-violet) and Tympanuchus

\section{Laboratory analyses}

We examined five non-MHC immune genes, which do not interact directly with pathogens (ChB6, IAP-1, IL-2, TGF- $\beta 3$, and TRAIL-like). ChB6 is a B cell surface antigen that induces a physiological signal for apoptosis in self-reactive lymphocytes, thus preventing autoimmune diseases in birds (Funk et al. 2003). IAP-1 is involved in antiapoptotic pathways including binding and inhibiting caspases and modulating receptor-mediated signal transduction (Yang and Li 2000). IL-2 plays a critical role in immune system function, as it induces the proliferation and differentiation of $\mathrm{T}, \mathrm{B}$, and natural killer (NK) (greater prairie-chicken T. cupido-orange, lesser prairie-chicken T. pallidicinctus - light blue, sharp-tailed chicken T. phasianellusgreen) grouse. Approximate locations of sampled populations are shown

cells (Zhou et al. 2001). TGF- $\beta 3$ belongs to a large family of cytokines which are cell-cell signaling proteins involved in the processes of proliferation, differentiation, growth, and migration of immune cells (Elliott and Blobe 2005). TRAIL-like cytokine stimulates apoptotic cell death and activates cytotoxic $\mathrm{T}$ cells, playing a role in the clearance of tumors and some viral infections (Falschlehner et al. 2009).

Amplifications of all five genes were performed using primer sets and annealing temperatures published for the domestic chicken (ChB6 and IAP-1, Zhou and Lamont 2003; IL-2, Zhou et al. 2001; TGF- $\beta 3$, Ye et al. 2006; TRAIL-like, Malek and Lamont 2003), and previously tested 
for the greater prairie-chicken (Bollmer et al. 2011). The following regions of the genes were amplified: (1) ChB6: exon encoding the extracellular part of the molecule; (2) IAP-1: exon encoding the BIR motif essential for gene function; (3) IL-2: promoter with a fragment of exon 1 ; (4) TGF- $\beta 3$ : intron 4 with a fragment of exon 5; and (5) TRAIL-like: intron 1 with a fragment of exon 1 (Table 1). The exon fragments amplified in the IL-2, TGF $\beta 3$, and TRAIL-like genes were small $(55-103 \mathrm{bp})$ with little sequence variation $(0-1$ SNP); therefore, we discarded these parts of the sequences and focused our analyses on the promoter (IL-2) and intron (TGF $\beta 3$ and TRAIL-like) regions. Analyses of diversity at exons were confined to ChB6 and IAP-1. All genes were amplified in $20-\mu 1$ polymerase chain reactions (PCRs) following methods described elsewhere (Bollmer et al. 2011). All PCR products were sequenced using Sanger sequencing technology in both forward and reverse directions at the University of Chicago Cancer Research Center DNA Sequencing Facility. Sequences not included among previously identified alleles (accession nos.: JN573105-JN573162) were deposited in GenBank (accession nos.: MF579259-MF579341). Each amplified gene region was considered orthologous, as haplotypes showed high nucleotide identity across all five grouse species (> 95\% identity), as well as with haplotypes derived from other galliform species (domestic chicken, Japanese quail Coturnix japonica, and turkey Meleagris gallopavo; > 90\% identity). There was no evidence for copy number variation of these genes in this or previous studies on the domestic chicken (Zhou et al. 2001, Malek and Lamont 2003, Zhou and Lamont 2003, Ye et al. 2006) and greater prairie-chicken (Bollmer et al. 2011).

\section{Analysis of diversity and selection}

All sequences were assembled, edited, and aligned in Geneious v7.1.7 (Biomatters Ltd., Auckland New Zealand). Sequences from each gene were assigned to haplotypes separately for each species using the PHASE algorithm (Stephens and Donnelly 2003) in DnaSP v5.0 (Librado and Rozas 2009). We used a burn-in of 1000 iterations, followed by 1000 iterations and a thinning interval of ten. Sequence polymorphism (total number of mutations and average number of nucleotide differences) across all five grouse species was analyzed with DnaSP v5.0.

We used MEGA v.6.0 (Tamura et al. 2013) to compare the fit of three basic nucleotide substitution models with variable base frequencies: (1) Hasegawa-Kishino-Yano (HKY) model, which assumes one transition rate and one transversion rate (Hasegawa et al. 1985); (2) Tamura-Nei (TN) model, which assumes variable transition rates and equal transversion rates (Tamura and Nei 1993); and (3) General Time Reversible (GTR) model, which assumes a symmetrical substitution matrix (Tavaré 1986). For both exon fragments, the HKY model showed the best fit, as indicated by the lowest corrected Akaike's Information Criterion (TN: $\triangle \mathrm{AIC}_{\mathrm{C}}=4.37$ for ChB6, $\Delta \mathrm{AIC}_{\mathrm{C}}=1.31$ for IAP-1; GTR: $\Delta \mathrm{AIC}_{\mathrm{C}}=11.33$ for ChB6, $\Delta \mathrm{AIC}_{\mathrm{C}}=4.62$ for IAP-1). Thus we used the HKY model in all further analyses. To test for positive and purifying selection on ChB6 and IAP-1 exon fragments, we assessed the relative rates of non-synonymous $(\mathrm{dN})$ and synonymous $(\mathrm{dS})$ nucleotide substitutions across the sequences (Hughes and Nei 1988). In general, a positive value for $\mathrm{dN}-\mathrm{dS}$ indicates an overabundance of nonsynonymous substitutions, which is consistent with positive selection, while the negative value indicates purifying selection. Codon-specific signatures of positive and purifying selection on ChB6 and IAP-1 exon fragments were tested using three different approaches implemented in the Datamonkey web server (Delport et al. 2010): single likelihood ancestor counting (SLAC), fixed effects likelihood (FEL), and random effects likelihood (REL). SLAC is considered to be the most conservative approach, while REL has been reported to suffer from higher rates of false positives for small data sets (Kosakovsky Pond \& Frost 2005). Under most scenarios, FEL approach is intermediate between SLAC and REL in terms of type I error (Kosakovsky Pond \& Frost 2005). We also used mixed effects model of evolution (MEME) to detect episodic diversifying selection affecting individual codon sites (Murrell et al. 2012).

\section{Phylogenetic analyses and haplotype networks}

Phylogenetic analyses were conducted using Bayesian inference (Markov Chain Monte Carlo method, MCMC) as implemented in MrBayes 3.2.6 (Huelsenbeck and Ronquist 2001). We specified the HKY model of nucleotide substitution. Four
Table 1 Polymorphism of five immune genes in Centrocercus and Tympanuchus grouse

\begin{tabular}{llllll}
\hline & ChB6 & IAP-1 & IL-2 & TGF- $\beta 3$ & TRAIL-like \\
\hline Fragment region & Exon 3 & Exon 2 & Promoter & Intron 4 & Intron 1 \\
Fragment size & $114 \mathrm{bp}$ & $323 \mathrm{bp}$ & $412 \mathrm{bp}$ & $685 \mathrm{bp}$ & $555 \mathrm{bp}$ \\
No. of variable sites & 6 & 7 & 14 & 24 & 34 \\
No. of haplotypes & 11 & 8 & 15 & 19 & 35 \\
$\begin{array}{l}\text { Average no. of nucleotide } \\
\quad \text { differences }\end{array}$ & $2.62 \pm 0.18$ & $1.93 \pm 0.35$ & $2.19 \pm 0.21$ & $4.80 \pm 0.63$ & $4.53 \pm 0.30$ \\
\hline
\end{tabular}


chains were set to run for 500,000 generations with a burn-in length of 100,000 . Trees were sampled every 100 generations for a total of 5000 trees. The initial 1000 sampled trees were discarded as the burn-in, and the $50 \%$ majority-rule Bayesian consensus trees and associated clade posterior probabilities were computed from the remaining 4000 trees. The domestic chicken Gallus gallus was used as the outgroup (GenBank accession nos.: X92865, ChB6; AF008592, IAP-1; AJ224516, IL-2; X60091, TGF- $\beta 3$; and AF537189, TRAIL-like). To further visualize differentiation of each gene and frequencies of phased haplotypes, we constructed median-joining haplotype networks (Bandelt et al. 1999) using the program Network v.4.6.10 (available at: www.fluxus-engineering.com).

\section{Results}

\section{Diversity and selection}

Each gene for all five grouse species had between six and 34 variable sites (Table 1), with a total of 85 variable nucleotide sites for all five immune genes. Most of the variable sites $(68 \%)$ were found within introns (TGF- $\beta 3$ and TRAIL-like). None of the variable sites were shared between Centrocercus and Tympanuchus genera (Fig. S1 in Electronic Supplementary Material). Haplotype reconstruction indicated the presence of 8 to 35 haplotypes per immune gene (Table 1), with a higher number of haplotypes observed for intron sequences $(61 \%)$. Introns were also associated with higher average nucleotide differences than the exons or the promoter sequences (Table 1). Among all five grouse species, $C$. minimus had the fewest haplotypes, both for exon and non-exon sequences $(n=7$ haplotypes in total), and both Centrocercus species had fewer haplotypes than observed in the Tympanuchus species (Table 2). T. cupido had the highest number of haplotypes (Table 2), but this was likely due to a much larger sample size $(n=247)$ when compared to each of the remaining species ( $n=28-30$, Table 2$)$.

We found little evidence for positive selection acting on ChB6 and IAP-1 genes. For ChB6, a signature of positive selection was detected for one codon (no. 13; Fig. S1 in ESM) using the REL method ( $p=0.01$ ), but this result was not confirmed with more conservative approaches (FEL: $p=0.25$, SLAC: $p=0.28$ ). No evidence for positive selection was found for the codons of the IAP-1 exon (all $p>0.05$ ). Purifying selection was detected with REL and FEL approaches for one ChB6 codon (no. 18; REL: $p<0.01$, FEL: $p=0.01$; Fig. S1 in ESM), but not for any of the IAP-1 codons (all $p>0.05$ ). Also, none of ChB6 and IAP-1 codons showed signatures of episodic diversifying selection with a MEME analysis (all $p>0.05$ ).

\section{Allele sharing and phylogenetic analyses}

No haplotypes were shared between Centrocercus and Tympanuchus genera. In contrast, there was a high rate of exon haplotype sharing among species within genera. Sixty-two percent of T. cupido exon haplotypes (ChB6 and IAP-1; $n=13$ ) were shared with other Tympanuchus species, and approximately half of those haplotypes $(n=7)$ were shared among all three Tympanuchus species. No private exon haplotypes were found in $T$. phasianellus or T. pallidicinctus (Table 2). The two C. minimus exon haplotypes were shared with C. urophasianus (Fig. 2).

Sharing of non-exon haplotypes was lower than with the exon haplotypes. Thirty-nine percent of $T$. cupido non-exon haplotypes (IL-2, TGF- $\beta 3$, and TRAIL-like; $n=38$ ) were shared with other Tympanuchus species and only two nonexon haplotypes (3\%) were shared among all three Tympanuchus species (Fig. 2). A total of 70 and $29 \%$ of non-exon haplotypes were private in $T$. phasianellus $(n=23)$ and $T$. pallidicinctus $(n=14)$, respectively (Table 2). Four out of the five $C$. minimus non-exon haplotypes were shared with $C$. urophasianus.

Four out of the five genes clustered by genus, as either Centrocercus or Tympanuchus haplotypes formed separate monophyletic clades (Figs. 2 and 3). The only exception was the IL-2 promoter gene, where two Centrocercus haplotypes formed a polytomy with one private $T$. phasianellus haplotype (Figs. 2 and 3). For the three genes (ChB6, IAP-1, and TGF- $\beta 3$ ) monophyletic Centrocercus clades had moderate or high (0.62-1.00) Bayesian posterior probabilities (Fig. 2), while for the TRAIL-like gene, Tympanuchus haplotypes formed a monophyletic clade with $100 \%$ Bayesian posterior probability (Fig. 2). For the ChB6 and IAP-1 exons, Centrocercus and Tympanuchus clades were connected by only one mutational step, while for the TGF- $\beta 3$ and TRAILlike introns, Centrocercus and Tympanuchus clades were several mutational steps apart (Fig. 3). In most genes (except for TGF- $\beta 3$ ), Tympanuchus haplotypes did not sort by species (Figs. 2 and 3). In contrast, all TGF- $\beta 3$ haplotypes of T. phasianellus formed a clade separated by three mutational steps from other Tympanuchus haplotypes (Figs. 2 and 3), supporting an earlier divergence of this species from the lineage leading to T. cupido and T. pallidicinctus.

\section{Discussion}

Our study revealed that several non-MHC immune genes showed extensive shared polymorphism within each of the two grouse genera, Centrocercus and Tympanuchus. At exons 
Table 2 Within-species polymorphism of five immune genes in Centrocercus and Tympanuchus grouse, where $n_{\mathrm{IND}}$ is the number of individuals genotyped, $n_{\mathrm{VS}}$ is the number of variable sites and $n_{\mathrm{HAP}}$ is the number of SNP haplotypes (number of private haplotypes $n_{\text {PRIV.HAP }}$ in parentheses)

\begin{tabular}{|c|c|c|c|c|c|c|c|}
\hline \multirow[t]{2}{*}{ Genus/species } & \multirow[t]{2}{*}{$n_{\mathrm{IND}}$} & \multicolumn{6}{|c|}{$n_{\mathrm{VS}} / n_{\mathrm{HAP}}\left(n_{\mathrm{PRIV} . \mathrm{HAP}}\right)$} \\
\hline & & ChB6 & IAP-1 & IL-2 & TGF- $\beta 3$ & TRAIL-like & Total \\
\hline \multicolumn{8}{|l|}{ Centrocercus } \\
\hline $\begin{array}{l}\text { C. minimus } \\
\text { (Gunnison sage-grouse) }\end{array}$ & 30 & $0 / 1(0)$ & $0 / 1(0)$ & $1 / 2(1)$ & $0 / 1(0)$ & $3 / 2(0)$ & $4 / 7(1)$ \\
\hline $\begin{array}{l}\text { C. urophasianus } \\
\text { (greater sage-grouse) }\end{array}$ & $29-30$ & $2 / 4(3)$ & $1 / 2(1)$ & $0 / 1(0)$ & $1 / 2(1)$ & $8 / 7(5)$ & $13 / 17(10)$ \\
\hline \multicolumn{8}{|l|}{ Tympanuchus } \\
\hline $\begin{array}{l}\text { T. phasianellus } \\
\text { (sharp-tailed grouse) }\end{array}$ & $28-30$ & $3 / 4(0)$ & $3 / 4(0)$ & $5 / 5(4)$ & 7/9 (9) & $7 / 9(3)$ & 25/31 (16) \\
\hline $\begin{array}{l}\text { T. pallidicinctus } \\
\text { (lesser prairie-chicken) }\end{array}$ & 30 & $3 / 4(0)$ & $2 / 3(0)$ & $5 / 6(2)$ & $1 / 2(1)$ & $5 / 6(1)$ & $16 / 21(4)$ \\
\hline $\begin{array}{l}\text { T. cupido } \\
\text { (greater prairie-chicken) }\end{array}$ & 247 & 4/7 (3) & $5 / 6(2)$ & $6 / 7(3)$ & $7 / 7(6)$ & $20 / 24(14)$ & $42 / 51(28)$ \\
\hline
\end{tabular}

of ChB6 and IAP-1, all allelic variants in T. phasianellus and T. pallidicinctus were shared with T. cupido, and a similar pattern was found within Centrocercus, in which all alleles of $C$. minimus were shared with $C$. urophasianus. While the rate of allele sharing for promoter (IL-2) and intron (TGF- $\beta 3$ and TRAIL-like) gene regions was lower, we did find several cases of non-exon allelic variants shared between species within genera (Fig. 2). In contrast, we found no evidence for allele sharing between genera. Allelic variants mostly sorted by genus, showing that ancestral polymorphism was not maintained following the divergence of Centrocercus and Tympanuchus genera.

Extensive shared polymorphism within Centrocercus and Tympanuchus grouse can be most likely explained by incomplete lineage sorting or introgression from hybridization. Species within each genus are thought to have diverged recently (within the last million years; Galla and Johnson 2015; Stein et al. 2015) and, thus, ancestral allelic lineages might have been retained in the descendant taxa, as there was not enough time for mutations to accumulate and achieve reciprocal monophyly. In most cases, shared genetic diversity resulting from incomplete lineage sorting is maintained for a relatively short period of time after species divergence. Under a simple allopatric speciation model, it takes roughly 9-12 $N_{e}$ (historical effective population size) generations to make incipient species reciprocally monophyletic at more than $95 \%$ of neutral nuclear loci (Hudson and Coyne 2002). Loci under directional selection are likely to require even shorter time to attain reciprocal monophyly, unless selection is counterbalanced by any retarding effects of population structure (Hudson and Coyne 2002). Finally, mitochondrial DNA becomes monophyletic more rapidly than a single nuclear gene, and far more rapidly than a sample of several nuclear genes. mtDNA also has greater potential for becoming monophyletic by selective sweeps and, thus, caution should be exercised when using mtDNA to infer genome-wide reciprocal monophyly in recently diverged taxa (Hudson and Coyne 2002). Despite this, molecular evidence for incomplete lineage sorting based on nuclear loci is relatively rare in birds (Rheindt et al. 2009; Qu et al. 2012).

The effects of incomplete lineage sorting are notoriously difficult to distinguish from recurrent gene flow, as both scenarios produce a similar pattern of allele sharing (Holder et al. 2001; Hedrick 2013). In our study system, intra-generic hybridization might be common in sympatric populations of grouse (Augustine and Trauba 2015), and female backcrosses may well result in introgression (Galla and Johnson 2015). Considering the limitations of our data (few nuclear loci genotyped), incomplete lineage sorting and introgression represent equally viable explanations for extensive shared polymorphisms found within Centrocercus and Tympanuchus grouse genera. In fact, molecular studies of birds have often suggested that the two mechanisms may operate at the same time in recently diverged populations or species (e.g., Milá et al. 2007; Campagna et al. 2010; Qu et al. 2012).

We found no evidence for shared polymorphism of the five non-MHC immune genes between Centrocercus and Tympanuchus grouse genera. In most cases, allelic variants sorted by genus, indicating that ancestral polymorphism was not maintained for long evolutionary periods in these taxa. Since none of the variable sites were shared between the two genera, there was no signature of convergent evolution within these immune genes. Also, we found no evidence for episodic diversifying selection acting on the ChB6 and IAP-1 exons, and no support for an excess of non-synonymous versus synonymous nucleotide substitutions in these sequences (as measured with $\mathrm{dN}$ and $\mathrm{dS}$ ), which could be indicative for balancing selection (Hughes and Nei 1988). These results are contrary to the patterns found at the MHC of Centrocercus and Tympanuchus grouse. There was evidence for allele sharing between the two grouse genera at both $\mathrm{MHC}$ classes I and II, although the strength of TSP was much 
ChB6

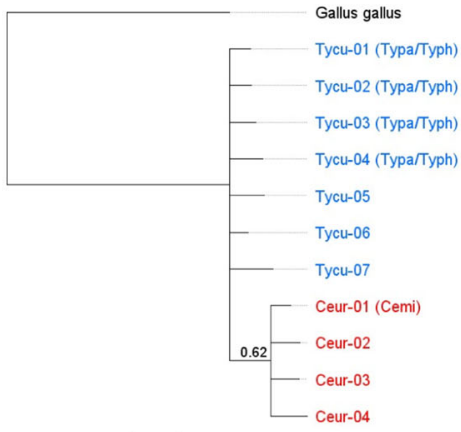

0.01

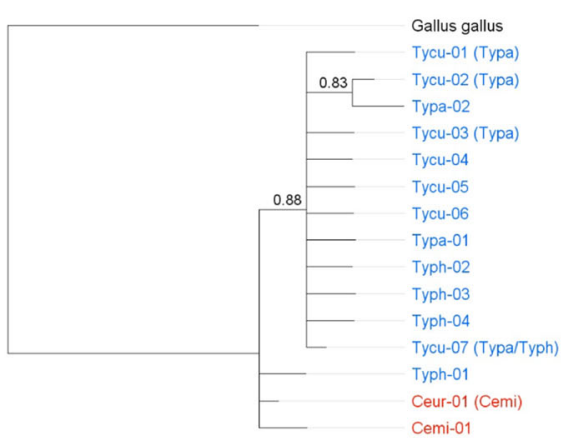

0.003

TGF-B3

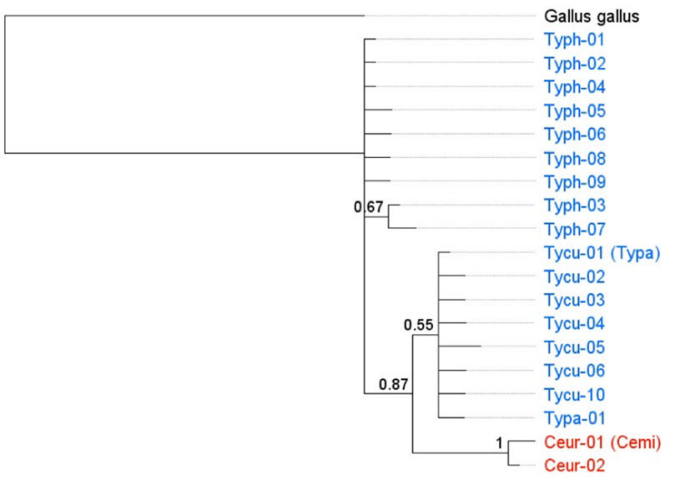

0.005

TRAIL-like

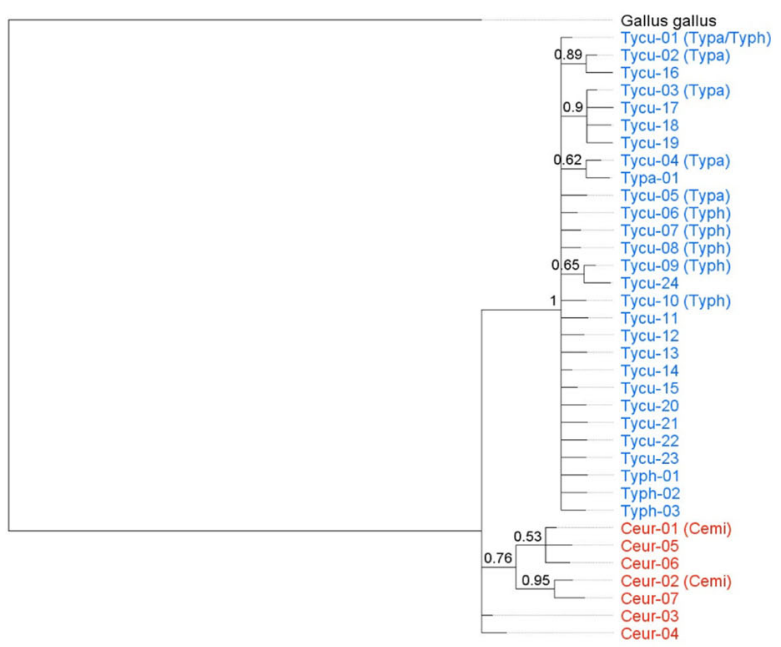

0.006
IAP-1

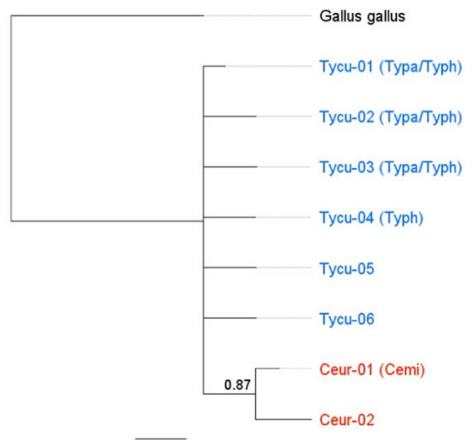

0.003

IL-2

Cemi-01 
Fig. 2 Bayesian 50\% majority-rule consensus trees obtained for five immune genes (exons: ChB6 and IAP-1; promoter: IL-2; introns: TGF$\beta 3$ and TRAIL-like) in Centrocercus (Cemi C. minimus, Ceur C. urophasianus) and Tympanuchus (Tycu T. cupido, Typa T. pallidicinctus, Typh T. phasianellus) grouse. Alleles shared between species are indicated by acronyms given in brackets. Centrocercus and Tympanuchus alleles are marked in red and blue, respectively. Weakly supported nodes are represented as unresolved. Bayesian posterior probabilities above $50 \%$ are indicated at the nodes. The domestic chicken Gallus gallus was used as the outgroup

weaker at MHC class I (Minias et al. 2016). This was consistent with contrasting patterns of selection at the two MHC classes in grouse, where class I was subject to much weaker diversifying and balancing selection (Minias et al. 2016). Although balancing selection may preserve MHC polymorphism for millions of years beyond species divergence (Ottová et al. 2005; Cutrera and Lacey 2007; Kamath and Getz 2011), the occurrence of balanced TSP at non-MHC immune genes seems to be scarce. A recent review by Téšický and Vinkler (2015) reported only a few non-MHC immune loci where shared polymorphisms could have been maintained by balancing selection. Some of the most notable examples include the oligoadenylate synthesase gene, which shows two deeply diverged allelic lineages predating the split of the house mouse Mus musculus and servant mouse Mus famulus 2.8 million years ago (Ferguson et al. 2008), and the immunoglobulin IgA hinge region, which shows balanced TSP in primates (Sumiyama et al. 2002).

In conclusion, our study provided evidence for extensive shared polymorphisms at non-MHC immune genes within two genera of North American prairie grouse, Centrocercus and Tympanuchus. This pattern is primarily attributable to introgression or incomplete lineage sorting following recent divergence and large ancestral effective population size (i.e., weak genetic drift). Our study suggests that prairie grouse may have attained relatively low degree of reciprocal monophyly at
Fig. 3 Median-joining haplotype networks for five immune genes (exons: ChB6 and IAP-1; promoter: IL-2; introns: TGF- $\beta 3$ and TRAIL-like) in Centrocercus and Tympanuchus grouse. Circles represent individual haplotypes, and the diameter of each circle corresponds to the number of samples with that particular haplotype. Tick marks between circles denote mutational steps required to connect haplotypes
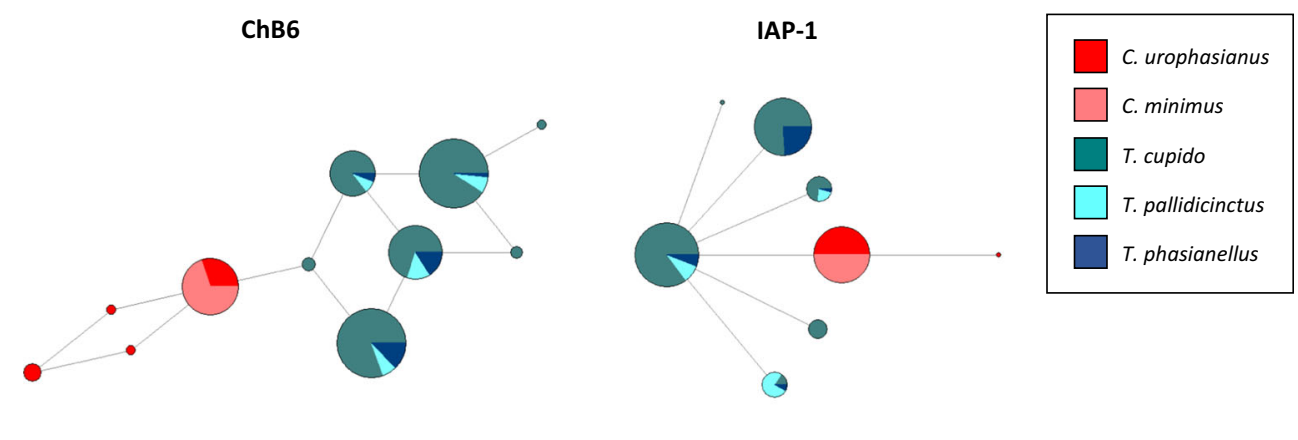

IL-2 TGF- $\beta 3$
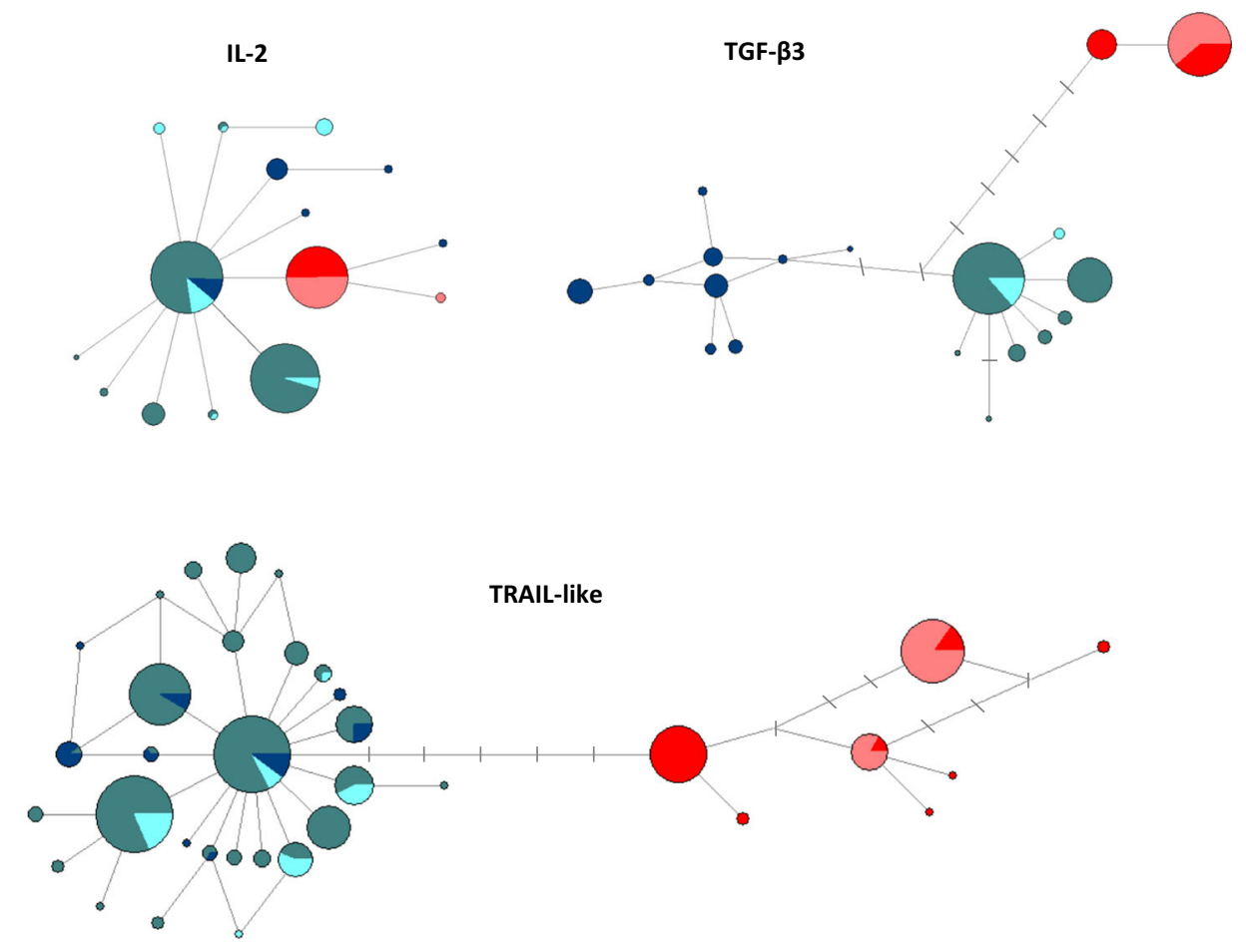
nuclear loci. The results also contrast with the patterns of balancing selection we previously found at the MHC of Centrocercus and Tympanuchus grouse (Minias et al. 2016) and reinforce the rarity of balancing selection in non-MHC immune genes.

Acknowledgements Funding was provided by grants from the Research Growth Initiative, University of Wisconsin-Milwaukee Graduate School, and National Science Foundation (DEB-0948695) to POD and LAW and a Fulbright Fellowship to PM. We thank A. Spaulding, D. Wolfe, and J. Toepfer for tissue collection. We thank two anonymous reviewers for helpful comments on the earlier drafts of the manuscript. Any use of trade, firm, or product names is for descriptive purposes only and does not imply endorsement by the U.S. Government.

Open Access This article is distributed under the terms of the Creative Commons Attribution 4.0 International License (http:// creativecommons.org/licenses/by/4.0/), which permits unrestricted use, distribution, and reproduction in any medium, provided you give appropriate credit to the original author(s) and the source, provide a link to the Creative Commons license, and indicate if changes were made.

\section{References}

Aguilar A, Garza JC (2007) Patterns of historical balancing selection on the salmonid major histocompatibility complex class II $\beta$ gene. J Mol Evol 65:34-43

Aldridge CL, Oyler-McCance SJ, Brigham RM (2001) Occurrence of greater sage-grouse $\mathrm{x}$ sharp-tailed grouse hybrids in Alberta. Condor 103:657-660

Augustine JK, Trauba DR (2015) Potential for behavioral reproductive isolation between greater prairie-chickens and sharp-tailed grouse in west-central Minnesota. J Ethol 33:25-24

Bain MR, Farley GH (2002) Apparent hybrid prairie-chickens in a zone of geographic overlap. Condor 104:683-687

Bandelt H-J, Forster P, Röhl A (1999) Median-joining networks for inferring intraspecific phylogenies. Mol Biol Evol 16:37-48

Bellinger MR, Johnson JA, Toepfer J, Dunn PO (2003) Loss of genetic variation in greater prairie chickens following a population bottleneck in Wisconsin, USA. Conserv Biol 17:717-724

Bollmer JL, Ruder EA, Johnson JA, Eimes JA, Dunn PO (2011) Drift and selection influence geographic variation at immune loci of prairiechickens. Mol Ecol 20:4695-4706

Campagna L, Lijtmaer DA, Kerr KCR, Barreira AS, Hebert PDN, Lougheed SC, Tubaro PL (2010) DNA barcodes provide new evidence of a recent radiation in the genus Sphorophila (Aves: Passeriformes). Mol Ecol Res 10:449-458

Cutrera AP, Lacey EA (2007) Trans-species polymorphism and evidence of selection on class II MHC loci in tuco-tucos (Rodentia: Ctenomyidae). Immunogenetics 59:937-948

Delport W, Poon AF, Frost SD, Kosakovsky Pond SL (2010) Datamonkey 2010: a suite of phylogenetic analysis tools for evolutionary biology. Bioinformatics 26:2455-2457

Donnelly MJ, Pinto J, Girod R, Besansky NJ, Lehmann T (2004) Revisiting the role of introgression vs shared ancestral polymorphisms as key processes shaping genetic diversity in the recently separated sibling species of the Anophles gambiae complex. Heredity 92:61-68

Drovetski SV (2003) Plio-Pleistocene climatic oscilations, Holarctic biogeography and speciation in an avian subfamily. J Biogeogr 30 : 1173-1181
Elliott RL, Blobe GC (2005) Role of transforming growth factor beta in human cancer. J Clin Oncol 23:2078-2093

Falschlehner C, Schaefer U, Walczak H (2009) Following TRAIL's path in the immune system. Immunology 127:145-154

Ferguson W, Dvora S, Gallo J, Orth A, Boissinot S (2008) Long-term balancing selection at the West Nile virus resistance gene, Oas $1 \mathrm{~b}$, maintains transspecific polymorphisms in the house mouse. Mol Biol Evol 25:1609-1618

Funk PE, Pifer J, Kharas M, Crisafi G, Johnson A (2003) The avian ChB6 alloantigen induces apoptosis in DT40 B cells. Cell Immunol 226: 95-104

Galla SJ, Johnson JA (2015) Differential introgression and effective size of marker type influence phylogenetic inference of a recently divergent avian group (Phasianidae: Tympanuchus). Mol Phylogenet Evol 84:1-13

Gilmour DG, Collins WM, Fredericksen TL, Urban WE Jr, Ward PF, DiFronzo NL (1986) Genetic interaction between non-MHC Tand B-cell alloantigens in response to Rous sarcomas in chickens. Immunogenetics 23:1-6

Grossen C, Keller L, Biebach I, Croll D, International Goat Genome Consortium (2014) Introgression from domestic goat generated variation at the major histocompatibility complex of alpine ibex. PLoS Genet 10:e1004438

Hasegawa M, Kishino H, Yano T (1985) Dating the human-age splitting by a molecular clock of mitochondrial DNA. J Mol Evol 22:160-174

Hedrick PW (2013) Adaptive introgression in animals: examples and comparison to new mutation and standing variation as sources of adaptive variation. Mol Ecol 22:4606-4618

Holder MT, Anderson JA, Holloway AK (2001) Difficulties in detecting hybridization. Syst Biol 50:978-982

Hudson RR, Coyne JA (2002) Mathematical consequences of the genealogical species concept. Evolution 56:1557-1565

Huelsenbeck JP, Ronquist F (2001) MRBAYES: Bayesian inference and phylogenetic trees. Bioinformatics 17:754-755

Hughes AL, Nei M (1988) Pattern of nucleotide substitution at major histocompatibility complex class I loci reveals overdominant selection. Nature 335:167-170

Kamath PL, Getz WM (2011) Adaptive molecular evolution of the major histocompatibility complex genes, DRA and DQA, in the genus Equus. BMC Evol Biol 11:128

Kosakovsky Pond SL, Frost SD (2005) Not so different after all: a comparison of methods for detecting amino acid sites under selection. Mol Biol Evol 22:1208-1222

Kriener K, O'hUigin C, Tichy H, Klein J (2000) Convergent evolution of major histocompatibility complex molecules in humans and new world monkeys. Immunogenetics 51:169-178

Leulier F, Lemaitre B (2008) Toll-like receptors - taking an evolutionary approach. Nat Rev Genet 9:165-178

Librado P, Rozas J (2009) DnaSP v5: a software for comprehensive analysis of DNA polymorphism data. Bioinformatics 25 : $1451-1452$

Liu W, Lamont SJ (2003) Candidate gene approach: potential associations of caspase-1, inhibitor of apoptosis protein-1, and prosaposin gene polymorphisms with response to Salmonella enteritidis challenge or vaccination in young chicks. Anim Biotechnol 14:61-76

Malek M, Lamont SJ (2003) Association of INOS, TRAIL, TGF- $\beta 2$, $T G F-\beta 3$, and $\operatorname{IgL}$ genes with response to Salmonella enteritidis in poultry. Genet Sel Evol 35:S99-S111

Milá B, Smith TB, Wayne RK (2007) Speciation and rapid phenotypic differentiation in the yellow-rumped warbler Dendroica coronate complex. Mol Ecol 16:159-173

Minias P, Bateson ZW, Whittingham LA, Johnson JA, Oyler-McCance S, Dunn PO (2016) Contrasting evolutionary histories of MHC class I 
and class II loci in grouse - effects of selection and gene conversion. Heredity 116:466-476

Murrell B, Wertheim JO, Moola S, Weighill T, Scheffler K, Kosakovsky Pond SL (2012) Detecting individual sites subject to episodic diversifying selection. PLoS Genet 8:e1002764

Nadachowska-Brzyska K, Zieliński P, Radwan J, Babik W (2012) Interspecific hybridization increases MHC class II diversity in two sister species of newts. Mol Ecol 21:887-906

Ottová E, Šimková A, Martin JF, De Bellocq JG, Gelnar M, Allienne JF, Morand S (2005) Evolution and trans-species polymorphism of MHC class II $\beta$ genes in cyprinid fish. Fish Shellfish Immunol 18: 199-222

Oyler-McCance SJ, DeYoung RW, Fike JA, Hagen CA, Johnson JA, Larsson LC, Patten MA (2016) Rangewide genetic analysis of lesser prairie-chicken reveals population structure, range expansion, and possible introgression. Conserv Genet 17:643-660

Oyler-McCance SJ, St John J, Taylor SE, Apa AD, Quinn TW (2005) Population genetics of Gunnison sage-grouse: implications for management. J Wildl Manag 69:630-637

Parmakelis A, Slotman MA, Marshall JC, Awono-Ambene PH, AntonioNkondijo C, Simard F, Caccone A, Powell JR (2008) The molecular evolution of four anti-malarial immune genes in the Anophles gambiae species complex. BMC Evol Biol 8:79

Persons NW, Hosner PA, Meiklejohn KA, Braun EL, Kimball RT (2016) Sorting out relationships among the grouse and ptarmigan using intron, mitochondrial, and ultra-conserved element sequences. Mol Phylogenet Evol 98:123-132

Qu Y, Zhang R, Quan Q, Song G, Li SH, Lei F (2012) Incomplete lineage sorting or secondary admixture: disentangling historical divergence from recent gene flow in the vinous-throated parrotbill (Paradoxornis webbianus). Mol Ecol 21:6117-6133

Rheindt FE, Christidis L, Norman JA (2009) Genetic introgression, incomplete lineage sorting and faulty taxonomy create multiple cases of polyphyly in a montane clade of tyrant-flycatchers (Elaenia, Tyrannidae). Zool Scr 38:143-153

Rheindt FE, Edwards SV (2011) Genetic introgression: an integral but neglected component of speciation in birds. Auk 128:620-632

Srithayakumar V, Castillo S, Mainguy J, Kyle CJ (2012) Evidence for evolutionary convergence at MHC in two broadly distributed mesocarnivores. Immunogenetics 64:289-301

Stein RW, Brown JW, Mooers AØ (2015) A molecular genetic time scale demonstrates cretaceous origins and multiple diversification rate shifts within the order Galliformes (Aves). Mol Phylogent Evol 95:155-164

Stephens M, Donelly P (2003) A comparison of bayesian methods for haplotype reconstruction from population genotype data. Am J Hum Genet 73:1162-1169

Sumiyama K, Saitou N, Ueda S (2002) Adaptive evolution of the IgA hinge region in primates. Mol Biol Evol 19:1093-1099

Tamura K, Nei M (1993) Estimation of the number of nucleotide substitutions in the control region of mitochondrial DNA in humans and chimpanzees. Mol Biol Evol 10:512-526

Tamura K, Stecher G, Peterson D, Filipski A, Kumar S (2013) MEGA6: molecular evolutionary genetics analysis version 6.0. Mol Biol Evol 30:2725-2729

Tavaré S (1986) Some probabilistic and statistical problems in the analysis of DNA sequences. In: Miura RM (ed) Some mathematical questions in biology-DNA sequence analysis. American Mathematical Society, Providence, Rhode Island, pp 57-86

Taylor RL Jr, Medarova Z, Briles WE (2016) Immune effects of chicken non-MHC alloantigens. Poult Sci 95:447-457

Téšický M, Vinkler M (2015) Trans-species polymorphism in immune genes: general pattern or MHC-restricted phenomenon? J Immunol Res 2015:838035

Yang YL, Li XM (2000) The IAP family: endogenous caspase inhibitors with multiple biological activities. Cell Res 10:169-177

Ye X, Avendano S, Dekkers JCM, Lamont SJ (2006) Association of twelve immune-related genes with performance of three broiler lines in two different hygiene environments. Poult Sci 85:1555-1569

Yeager M, Kumar S, Hughes AL (1997) Sequence convergence in the peptide-binding region of primate and rodent MHC class Ib molecules. Mol Evol Biol 14:1035-1041

Young JR, Braun CE, Oyler-McCance SJ, Aldridge CL, Magee PA, Schroeder MA (2015) Gunnison Sage-Grouse. In: The birds of North America, vol 721, pp 1-27

Zhou H, Lamont SJ (2003) Associations of six candidate genes with antibody response kinetics in hens. Poult Sci 82:1118-1126

Zhou H, Buitenhuis AJ, Weigend S, Lamont SJ (2001) Candidate gene promoter polymorphism and antibody response kinetics in chickens: interferon- $\gamma$, interleukin-2, and immunoglobulin light chain. Poult Sci 80:1679-1689

Zhou Y, Duvaux L, Ren G, Zhang L, Savolainen O, Liu J (2017) Importance of incomplete lineage sorting and introgression in the origin of shared genetic variation between two closely related pines with overlapping distributions. Heredity 118:211-220 